\title{
Numerical Analysis of the Magnetization Behavior in Magnetic Resonance Imaging in the Presence of Multiple Chemical Exchange Pools
}

\author{
Kenya Murase \\ Department of Medical Physics and Engineering, Division of Medical Technology and Science, Faculty of Health Science, \\ Graduate School of Medicine, Osaka University, Osaka, Japan \\ Email: murase@sahs.med.osaka-u.ac.jp
}

How to cite this paper: Murase, K. (2017) Numerical Analysis of the Magnetization Behavior in Magnetic Resonance Imaging in the Presence of Multiple Chemical Exchange Pools. Open Journal of Applied Sciences, 7, 1-14.

http://dx.doi.org/10.4236/ojapps.2017.71001

Received: December 16, 2016

Accepted: January 10, 2017

Published: January 13, 2017

Copyright $\odot 2017$ by author and Scientific Research Publishing Inc. This work is licensed under the Creative Commons Attribution International License (CC BY 4.0).

http://creativecommons.org/licenses/by/4.0/

\begin{abstract}
The purpose of this study was to demonstrate a simple and fast method for solving the time-dependent Bloch-McConnell equations describing the behavior of magnetization in magnetic resonance imaging (MRI) in the presence of multiple chemical exchange pools. First, the time-dependent BlochMcConnell equations were reduced to a homogeneous linear differential equation, and then a simple equation was derived to solve it using a matrix operation and Kronecker tensor product. From these solutions, the longitudinal relaxation rate $\left(R_{1 \rho}\right)$ and transverse relaxation rate in the rotating frame $\left(R_{2 \rho}\right)$ and $\mathrm{Z}$-spectra were obtained. As illustrative examples, the numerical solutions for linear and star-type three-pool chemical exchange models and linear, startype, and kite-type four-pool chemical exchange models were presented. The effects of saturation time (ST) and radiofrequency irradiation power $\left(\omega_{1}\right)$ on the chemical exchange saturation transfer (CEST) effect in these models were also investigated. Although $R_{1 \rho}$ and $R_{2 \rho}$ were not affected by the ST, the CEST effect observed in the Z-spectra increased and saturated with increasing ST. When $\omega_{1}$ was varied, the CEST effect increased with increasing $\omega_{1}$ in $R_{1 \rho}, R_{2 \rho}$, and Z-spectra. When $\omega_{1}$ was large, however, the spillover effect due to the direct saturation of bulk water protons also increased, suggesting that these parameters must be determined in consideration of both the CEST and spillover effects. Our method will be useful for analyzing the complex CEST contrast mechanism and for investigating the optimal conditions for CEST MRI in the presence of multiple chemical exchange pools.
\end{abstract}

\section{Keywords}

Bloch-McConnell Equations, Multiple Chemical Exchange Pools, Chemical Exchange Saturation Transfer (CEST) Magnetic Resonance Imaging (MRI), Amide Proton Transfer (APT) MRI, Numerical Analysis 


\section{Introduction}

Chemical exchange saturation transfer (CEST) is a novel contrast mechanism for magnetic resonance imaging (MRI) [1] and has been increasingly used to detect dilute proteins via the interaction between labile solute protons and bulk water protons [2] [3] [4]. Moreover, amide proton transfer (APT) imaging, a particular type of CEST MRI that specifically probes labile amide protons of endogenous mobile proteins and peptides in tissue, has been explored for imaging diseases such as acute stroke and tumor, and is currently under intensive evaluation for clinical translation [5] [6]. Furthermore, various CEST agents have been actively developed to detect the parameters that reflect tissue $\mathrm{pH}$ and molecular environment and/or to enhance the CEST effect [7]. However, CEST or APT MRI contrast mechanism is complex, depending on not only the concentration of CEST agents or amide protons, exchange and relaxation properties, but also varying with experimental conditions such as magnetic field strength and radiofrequency (RF) power [8]. When there are multiple exchangeable sites within a single CEST system, the CEST contrast mechanism becomes even more complex [9]. Thus, in analyzing the complex CEST contrast mechanism and for investigating the optimal study conditions, numerical simulations are useful and effective [10] [11]. To perform extensive numerical simulations for CEST or APT MRI, it will be necessary to develop a simple and fast method for obtaining the numerical solutions to the time-dependent Bloch-McConnell equations.

The purpose of this study was to present a simple and fast method for solving the time-dependent Bloch-McConnell equations for analyzing the behavior of magnetization in MRI in the presence of multiple chemical exchange pools.

\section{Materials and Methods}

\subsection{Bloch-McConnell Equations in a Two-Pool Chemical Exchange Model}

Figure 1 illustrates a two-pool chemical exchange model in which pool $A$ represents the bulk water pool. The time-dependent Bloch-McConnell equations in the two-pool exchange model for CEST MRI are given by [10] [11]

$$
\left\{\begin{array}{l}
\frac{\mathrm{d} M_{x}^{a}(t)}{\mathrm{d} t}=-R_{2}^{a} M_{x}^{a}(t)-k_{a b} M_{x}^{a}(t)+\Delta \omega_{a} M_{y}^{a}(t)+k_{b a} M_{x}^{b}(t) \\
\frac{\mathrm{d} M_{y}^{a}(t)}{\mathrm{d} t}=-\Delta \omega_{a} M_{x}^{a}(t)-R_{2}^{a} M_{y}^{a}(t)-k_{a b} M_{y}^{a}(t)+\omega_{1} M_{z}^{a}(t)+k_{b a} M_{y}^{b}(t) \\
\frac{\mathrm{d} M_{z}^{a}(t)}{\mathrm{d} t}=-\omega_{1} M_{y}^{a}(t)-R_{1}^{a} M_{z}^{a}(t)-k_{a b} M_{z}^{a}(t)+k_{b a} M_{z}^{b}(t)+R_{1}^{a} M_{0}^{a} \\
\frac{\mathrm{d} M_{x}^{b}(t)}{\mathrm{d} t}=k_{a b} M_{x}^{a}(t)-k_{b a} M_{x}^{b}(t)-R_{2}^{b} M_{x}^{b}(t)+\Delta \omega_{b} M_{y}^{b}(t) \\
\frac{\mathrm{d} M_{y}^{b}(t)}{\mathrm{d} t}=k_{a b} M_{y}^{a}(t)-\Delta \omega_{b} M_{x}^{b}(t)-R_{2}^{b} M_{y}^{b}(t)-k_{b a} M_{y}^{b}(t)+\omega_{1} M_{z}^{b}(t) \\
\frac{\mathrm{d} M_{z}^{b}(t)}{\mathrm{d} t}=k_{a b} M_{z}^{a}(t)-\omega_{1} M_{y}^{b}(t)-R_{1}^{b} M_{z}^{b}(t)-k_{b a} M_{z}^{b}(t)+R_{1}^{b} M_{0}^{b}
\end{array}\right.
$$




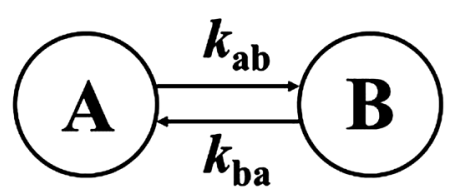

Figure 1. Illustration of a two-pool chemical exchange model. $k_{a b}$ and $k_{b a}$ represent the exchange rates from pool $A$ to pool $B$ and from pool $B$ to pool $A$, respectively.

where superscripts $a$ and $b$ show the parameters in pool $A$ and pool $B$, respectively. For example, $M_{x}^{a}(t), M_{y}^{a}(t)$, and $M_{z}^{a}(t)$ denote the $x, y$, and $z$ components of the magnetization in the rotating frame in pool $A$ at time $t$, respectively. $R_{1}^{a}$ and $R_{2}^{a}$ denote the longitudinal and transverse relaxation rates, i.e., the reciprocals of the longitudinal $\left(T_{1}^{a}\right)$ and transverse relaxation times $\left(T_{2}^{a}\right)$ in pool $A$, respectively. $k_{a b}$ denotes the exchange rate from spins in pool $A$ to those in pool $B$, whereas $k_{b a}$ denotes that from spins in pool $B$ to those in pool A. $\quad M_{0}^{a}$ and $M_{0}^{b}$ denote the thermal equilibrium $z$ magnetizations in pool $A$ and pool $B$, respectively. $\Delta \omega_{a}$ and $\Delta \omega_{b}$ are given by $\omega_{a}-\omega$ and $\omega_{b}-\omega$, respectively, where $\omega_{a}$ and $\omega_{b}$ are the Larmor frequencies in pool $A$ and pool $B$, respectively, and $\omega$ is the frequency of the RF irradiation applied along the $x$ axis of the rotating frame. $\omega_{1}$ is the amplitude of the RF irradiation.

The differential equations given by Equation (1) can be combined into one vector equation (homogeneous linear differential equation) [11]:

$$
\frac{\mathrm{d} \boldsymbol{M}}{\mathrm{d} t}=\boldsymbol{A} \cdot \boldsymbol{M},
$$

where

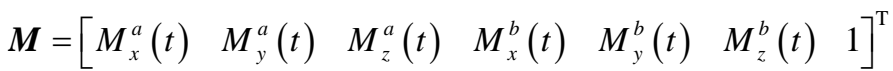

and

$$
\boldsymbol{A}=\left[\begin{array}{cccc}
-\left(R_{2}^{a}+k_{a b}\right) & \Delta \omega_{a} & 0 & k_{b a} \\
-\Delta \omega_{a} & -\left(R_{2}^{a}+k_{a b}\right) & \omega_{1} & 0 \\
0 & -\omega_{1} & -\left(R_{1}^{a}+k_{a b}\right) & 0 \\
k_{a b} & 0 & 0 & -\left(R_{2}^{b}+k_{b a}\right) \\
0 & k_{a b} & 0 & -\Delta \omega_{b} \\
0 & 0 & k_{a b} & 0 \\
0 & 0 & 0 & 0 \\
0 & 0 & 0 \\
k_{b a} & 0 & 0 \\
0 & k_{b a} & R_{1}^{a} M_{0}^{a} \\
\Delta \omega_{b} & 0 & 0 \\
-\left(R_{2}^{b}+k_{b a}\right) & \omega_{1} & 0 \\
-\omega_{1} & -\left(R_{1}^{b}+k_{b a}\right) & R_{1}^{b} M_{0}^{b} \\
0 & 0 & 0
\end{array}\right],
$$

where $\mathrm{T}$ in Equation (3) denotes the matrix transpose. According to Koss et al. 
[12], the matrix $A$ can be given by

$$
\boldsymbol{A}=\left[\begin{array}{cc}
\boldsymbol{E} & \boldsymbol{C} \\
0 & 0
\end{array}\right]
$$

where $E$ is the evolution matrix [12] and $C$ is the constant-term matrix. Furthermore, $E$ is given by

$$
\boldsymbol{E}=\boldsymbol{R}+\boldsymbol{K} .
$$

In the case of $\boldsymbol{A}$ given by Equation (4), $R$ is reduced to

$$
\boldsymbol{R}=\left[\begin{array}{cc}
\boldsymbol{R}^{a} & 0 \\
0 & \boldsymbol{R}^{b}
\end{array}\right]
$$

where

$$
\boldsymbol{R}^{a}=\left[\begin{array}{ccc}
-R_{2}^{a} & \Delta \omega_{a} & 0 \\
-\Delta \omega_{a} & -R_{2}^{a} & \omega_{1} \\
0 & -\omega_{1} & -R_{1}^{a}
\end{array}\right]
$$

and

$$
\boldsymbol{R}^{b}=\left[\begin{array}{ccc}
-R_{2}^{b} & \Delta \omega_{b} & 0 \\
-\Delta \omega_{b} & -R_{2}^{b} & \omega_{1} \\
0 & -\omega_{1} & -R_{1}^{b}
\end{array}\right]
$$

$K$ in Equation (6) is given by

$$
\boldsymbol{K}=\left[\begin{array}{cc}
-k_{a b} & k_{b a} \\
k_{a b} & -k_{b a}
\end{array}\right] \otimes \boldsymbol{I},
$$

where $I$ is a 3-by-3 identity matrix and $\otimes$ denotes the Kronecker tensor product. $C$ in Equation (5) is given by

$$
\boldsymbol{C}=\left[\begin{array}{ll}
R_{1}^{a} M_{0}^{a} & R_{1}^{b} M_{0}^{b}
\end{array}\right]^{\mathrm{T}} \otimes\left[\begin{array}{lll}
0 & 0 & 1
\end{array}\right]^{\mathrm{T}} .
$$

The solution of Equation (2) can be given by [11]

$$
\boldsymbol{M}(t)=e^{\boldsymbol{A t}} \boldsymbol{M}(0),
$$

where $t$ represents the so-called saturation time and $\boldsymbol{M}(0)$ is the matrix of initial values at $t=0 . e^{A t}$ is the matrix exponential.

\subsection{Linear Three-Pool Chemical Exchange Model}

Figure 2(a) illustrates a linear three-pool chemical exchange model in which pool $A$ represents the bulk water pool. In this case, $R$ and $K$ are given by [12]

$$
\boldsymbol{R}=\left[\begin{array}{ccc}
\boldsymbol{R}^{a} & 0 & 0 \\
0 & \boldsymbol{R}^{b} & 0 \\
0 & 0 & \boldsymbol{R}^{c}
\end{array}\right]
$$

and

$$
\boldsymbol{K}=\left[\begin{array}{ccc}
-k_{a b}-k_{a c} & k_{b a} & k_{c a} \\
k_{a b} & -k_{b a} & 0 \\
k_{a c} & 0 & -k_{c a}
\end{array}\right] \otimes \boldsymbol{I},
$$




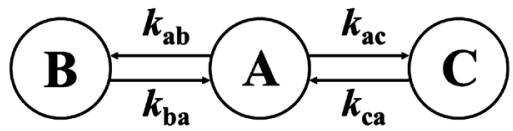

(a)

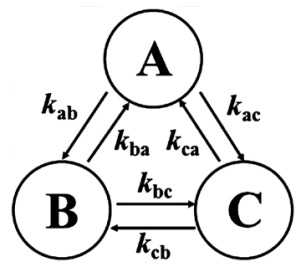

(b)

Figure 2. Illustration of three-pool chemical exchange models. (a) and (b) show linear and star-type three-pool chemical exchange models, respectively. As in the case of $k_{a b}, k_{a c}$ $k_{c a}, k_{b c}$ and $k_{c b}$ represent the exchange rates from pool $A$ to pool $C$, from pool $C$ to pool $A$, from pool $B$ to pool $C$, and from pool $C$ to pool $B$, respectively.

respectively. $\boldsymbol{R}^{c}$ in Equation (13) is given by Equation (8) in which the subscript $a$ and superscript $a$ are replaced by $c$. $C$ is given by

$$
\boldsymbol{C}=\left[\begin{array}{lll}
R_{1}^{a} M_{0}^{a} & R_{1}^{b} M_{0}^{b} & R_{1}^{c} M_{0}^{c}
\end{array}\right]^{\mathrm{T}} \otimes\left[\begin{array}{lll}
0 & 0 & 1
\end{array}\right]^{\mathrm{T}} .
$$

\subsection{Triangular Three-Pool Chemical Exchange Model}

Figure 2(b) illustrates a triangular three-pool chemical exchange model in which pool $A$ represents the bulk water pool. In this case, $K$ is given by [12]

$$
\boldsymbol{K}=\left[\begin{array}{ccc}
-k_{a b}-k_{a c} & k_{b a} & k_{c a} \\
k_{a b} & -k_{b a}-k_{b c} & k_{c b} \\
k_{a c} & k_{b c} & -k_{c a}-k_{c b}
\end{array}\right] \otimes \boldsymbol{I} .
$$

\subsection{Linear Four-Pool Chemical Exchange Model}

Figure 3(a) illustrates a linear four-pool chemical exchange model in which pool $A$ represents the bulk water pool. In this case, $R$ and $K$ are given by [12]

$$
\boldsymbol{R}=\left[\begin{array}{cccc}
\boldsymbol{R}^{a} & 0 & 0 & 0 \\
0 & \boldsymbol{R}^{b} & 0 & 0 \\
0 & 0 & \boldsymbol{R}^{c} & 0 \\
0 & 0 & 0 & \boldsymbol{R}^{d}
\end{array}\right]
$$

and

$$
\boldsymbol{K}=\left[\begin{array}{cccc}
-k_{a b}-k_{a c} & k_{b a} & k_{c a} & 0 \\
k_{a b} & -k_{b a} & 0 & 0 \\
k_{a c} & 0 & -k_{c a}-k_{c d} & k_{d c} \\
0 & 0 & k_{c d} & -k_{d c}
\end{array}\right] \otimes \boldsymbol{I},
$$

respectively. $\boldsymbol{R}^{d}$ in Equation (17) is given by Equation (8) in which the subscript $a$ and superscript $a$ are replaced by $d$. $C$ is given by

$$
\boldsymbol{C}=\left[\begin{array}{llll}
R_{1}^{a} M_{0}^{a} & R_{1}^{b} M_{0}^{b} & R_{1}^{c} M_{0}^{c} & R_{1}^{d} M_{0}^{d}
\end{array}\right]^{\mathrm{T}} \otimes\left[\begin{array}{lll}
0 & 0 & 1
\end{array}\right]^{\mathrm{T}} .
$$

\subsection{Star-Type Four-Pool Chemical Exchange Model}

Figure 3(b) illustrates a star-type four-pool chemical exchange model in which pool $A$ represents the bulk water pool. In this case, $K$ is given by [12]. 


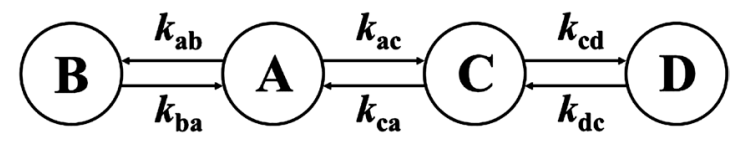

(a)

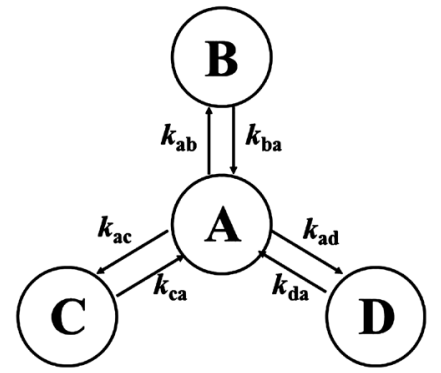

(b)

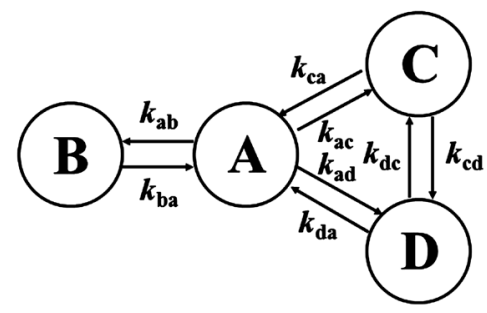

(c)

Figure 3. Illustration of four-pool chemical exchange models. (a), (b), and (c) show linear, star-type, and kite-type four-pool chemical exchange models, respectively. As in the case of $k_{a b}, k_{a d} k_{d a}, k_{c d}$ and $k_{d c}$ represent the exchange rates from pool $A$ to pool $D$, from pool $D$ to pool $A$, from pool $C$ to pool $D$, and from pool $D$ to pool $C$, respectively.

$$
\boldsymbol{K}=\left[\begin{array}{cccc}
-k_{a b}-k_{a c}-k_{a d} & k_{b a} & k_{c a} & k_{d a} \\
k_{a b} & -k_{b a} & 0 & 0 \\
k_{a c} & 0 & -k_{c a} & 0 \\
k_{a d} & 0 & 0 & -k_{d a}
\end{array}\right] \otimes \boldsymbol{I} .
$$

\subsection{Kite-Type Four-Pool Chemical Exchange Model}

Figure 3(c) illustrates a kite-type four-pool chemical exchange model in which pool $A$ represents the bulk water pool. Although there are no chemical exchanges between pool $C$ and pool $D$ in the star-type four-pool chemical exchange model (Figure $3(\mathrm{~b})$ ), there are exchanges between them in the kite-type model (Figure $3(\mathrm{c}))$. In this case, $K$ is given by [12]

$$
\boldsymbol{K}=\left[\begin{array}{cccc}
-k_{a b}-k_{a c}-k_{a d} & k_{b a} & k_{c a} & k_{d a} \\
k_{a b} & -k_{b a} & 0 & 0 \\
k_{a c} & 0 & -k_{c a}-k_{c d} & k_{d c} \\
k_{a d} & 0 & k_{c d} & -k_{d a}-k_{d c}
\end{array}\right] \otimes \boldsymbol{I} .
$$

It should be noted that mass balance imposes the following relationship between the exchange rates $\left(k_{i j}\right.$ and $\left.k_{j i}\right)$ of pool $I$ and pool $J[10]$ :

$$
k_{i j}=\left(k_{i j}+k_{j i}\right) \cdot \frac{M_{0}^{j}}{M_{0}^{i}+M_{0}^{j}}
$$

and

$$
k_{j i}=\left(k_{i j}+k_{j i}\right) \cdot \frac{M_{0}^{i}}{M_{0}^{i}+M_{0}^{j}}
$$

\subsection{Calculation of $R_{1 \rho}, R_{2 \rho}$, and Z-Spectra}

The longitudinal relaxation rate in the rotating frame $\left(R_{1 \rho}\right)$ was obtained from the negative of the largest (least negative) real eigenvalue $\left(\lambda_{1}\right)$ of the matrix $A$ 
in Equation (2), i.e., $R_{1 \rho}=-\lambda_{1}$ [12] [13].

The transverse relaxation rate in the rotating frame $\left(R_{2 \rho}\right)$ was obtained from the absolute value of the largest real part of the complex eigenvalue $\left(\lambda_{2}\right)$ of the matrix $\boldsymbol{A}$ in Equation (2), i.e., $R_{2 \rho}=\left|\operatorname{Re}\left(\lambda_{2}\right)\right|$ [13].

The CEST effect has usually been analyzed using the so-called Z-spectrum [11]. Thus, we calculated the Z-spectrum by using the following equation:

$$
\mathrm{Z}-\text { spectrum }=\frac{M_{\mathrm{z}}^{a}\left(\Delta \omega_{\mathrm{off}}\right)}{M_{0}^{a}},
$$

where $M_{z}^{a}\left(\Delta \omega_{\text {off }}\right)$ denotes the $z$ magnetization of pool $A$ (bulk water) with saturation at $\Delta \omega_{\text {off }}$. It should be noted that $\Delta \omega_{\text {off }}$ is the offset frequency of the RF irradiation from the Larmor frequency of bulk water protons, i.e., $\Delta \omega_{\text {off }}=-\Delta \omega_{a}$. In this study, $\Delta \omega_{\text {off }}$ was varied from $-3000 \mathrm{~Hz}$ to $3000 \mathrm{~Hz}$ with an interval of $100 \mathrm{~Hz}$.

\subsection{Simulation Studies}

Because we have already treated a two-pool chemical exchange model in our previous paper [11], we treated three-pool and four-pool chemical exchange models in this study.

First, we considered the three-pool exchange model consisting of bulk water (pool $A$ ) and two labile proton pools (pool $B$ and pool $C$ ) as illustrative examples. In this case, we assumed that the longitudinal $\left(T_{1}^{a}\right)$ and transverse relaxation times for bulk water $\left(T_{2}^{a}\right)$ were $3 \mathrm{~s}$ and $100 \mathrm{~ms}$, respectively, and were $1 \mathrm{~s}$ and $15 \mathrm{~ms}$ for two labile protons, i.e., $T_{1}^{b}=T_{1}^{c}=1 \mathrm{~s}$ and $T_{2}^{b}=T_{2}^{c}=15 \mathrm{~ms}$ [9]. The chemical shifts for two labile protons were set to be $4 \mathrm{ppm}$ and $5 \mathrm{ppm}$. It should be noted that the chemical shifts of $4 \mathrm{ppm}$ and $5 \mathrm{ppm}$ correspond to $\Delta \omega_{\text {off }}$ of $1192.8 \mathrm{~Hz}$ and $1491.0 \mathrm{~Hz}$, respectively, for the magnetic field strength of $7 \mathrm{~T}$. Unless specifically stated, $k_{a b}+k_{b a}, k_{a c}+k_{c a}$, and $k_{b c}+k_{c b}$ were assumed to be $100 \mathrm{~Hz}, 300 \mathrm{~Hz}$, and $100 \mathrm{~Hz}$, respectively. $M_{0}^{a}, M_{0}^{b}$, and $M_{0}^{c}$ were assumed to be $1,1 / 250$, and $1 / 500$, respectively. The saturation time and $\omega_{1}$ were taken as $5 \mathrm{~s}$ and $50 \mathrm{~Hz}$, respectively.

For four-pool exchange models, we simulated one nuclear Overhauser effect site (pool $D$ ) in addition to the above bulk water (pool $A$ ) and two labile proton pools (pool $B$ and pool $C$ ). We assumed that the longitudinal $\left(T_{1}^{d}\right)$ and transverse relaxation times for pool $D\left(T_{2}^{d}\right)$ were $1 \mathrm{~s}$ and $5 \mathrm{~ms}$, respectively [9]. The chemical shift for pool $D$ was set to be $-3.5 \mathrm{ppm}$. It should be noted that the chemical shift of $-3.5 \mathrm{ppm}$ corresponds to $\Delta \omega_{\text {off }}$ of $-1043.7 \mathrm{~Hz}$ for the magnetic field strength of $7 \mathrm{~T}$. Unless specifically stated, $k_{a d}+k_{d a}$ and $k_{c d}+k_{d c}$ were assumed to be $10 \mathrm{~Hz}$ and $10 \mathrm{~Hz}$, respectively. $M_{0}^{d}$ was assumed to be $1 / 500$.

Calculations were performed using MATLAB $^{\circledast}$ (The MathWorks Inc., Natick, MA, USA) on an Intel Core ${ }^{\mathrm{TM}} \mathrm{i} 7-4790 \mathrm{CPU}(3.6 \mathrm{GHz})$ with 8-GB RAM. The matrix exponential and Kronecker tensor product were calculated using the MATLAB ${ }^{\circledast}$ functions “expm" and "kron", respectively. 


\section{Results}

Figure 4 shows the $R_{1 \rho}$ (a), $R_{2 \rho}$ (b), and Z-spectra (c) as a function of offset frequency $\left(\Delta \omega_{\text {off }}\right)$ for various saturation times $(0.5,1,2,5$, and $10 \mathrm{~s})$ in the linear three-pool chemical exchange model (Figure 2(a)). It should be noted that the common logarithm of the $R_{1 \rho}$ value was plotted in Figure 4(a) in order to enlarge the change in the $R_{1 \rho}$ value. The peaks at $0 \mathrm{~Hz}(0 \mathrm{ppm}), 1192.8 \mathrm{~Hz}(4$ ppm), and $1491.0 \mathrm{~Hz}(5 \mathrm{ppm})$ derive from pool $A$, pool $B$, and pool $C$, respectively. As shown in Figure $4, R_{1 \rho}$ and $R_{2 \rho}$ were not affected by the saturation time, whereas Z-spectra changed largely depending on the saturation time, i.e., $Z$-spectra became broad and tended to saturate with increasing saturation time.

Figure 5 shows the $R_{1 \rho}$ (a), $R_{2 \rho}$ (b), and Z-spectra (c) as a function of $\Delta \omega_{\text {off }}$ for various $\omega_{1}$ values $(25,50,100,150$, and $200 \mathrm{~Hz})$ in the linear threepool chemical exchange model (Figure 2(a)). As in Figure 4, the peaks at $0 \mathrm{~Hz}(0$ ppm), $1192.8 \mathrm{~Hz}$ (4 ppm), and $1491.0 \mathrm{~Hz}$ (5 ppm) derive from pool $A$, pool $B$, and pool $C$, respectively. As shown in Figure 5, all parameters became broad with increasing $\omega_{1}$ value.

Figure 6 shows the $R_{1 \rho}$ (a), $R_{2 \rho}$ (b), and Z-spectra (c) as a function of $\Delta \omega_{\text {off }}$ for various $M_{0}^{c}$ values $(1 / 500,1 / 250,1 / 125,1 / 100$, and 1/50) in the triangular three-pool chemical exchange model (Figure 2(b)). As shown in Figure 6(a) and Figure 6(c), the peaks at $1491.0 \mathrm{~Hz}(5 \mathrm{ppm})$ derived from pool $C$ changed largely depending on $M_{0}^{c}$ in both $R_{1 \rho}$ and Z-spectra. The peaks at $1192.8 \mathrm{~Hz}$ (4 ppm) derived from pool $B$ also changed but to a lesser extent. As shown in Figure 6(b), the $R_{2 \rho}$ values increased with increasing $M_{0}^{c}$ value.

Figure 7 shows the $R_{1 \rho}$ (a), $R_{2 \rho}$ (b), and Z-spectra (c) as a function of $\Delta \omega_{\text {off }}$ for various $\omega_{1}$ values $(25,50,100,150$, and $200 \mathrm{~Hz})$ in the linear fourpool chemical exchange model (Figure 3(a)). When $\omega_{1}$ was small, four peaks were clearly observed at $0 \mathrm{~Hz}(0 \mathrm{ppm}), 1192.8 \mathrm{~Hz}(4 \mathrm{ppm}), 1491.0 \mathrm{~Hz}(5 \mathrm{ppm})$,

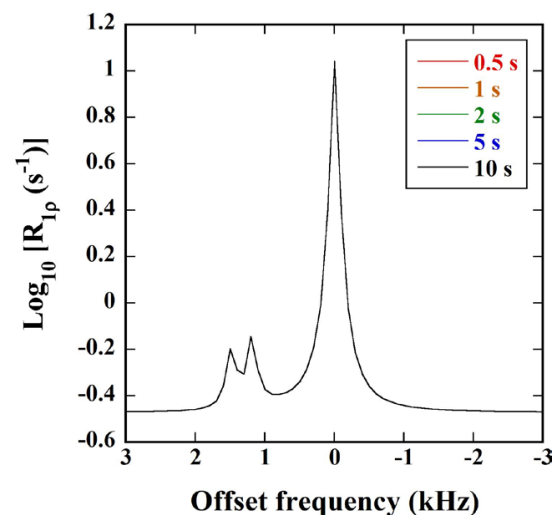

(a)

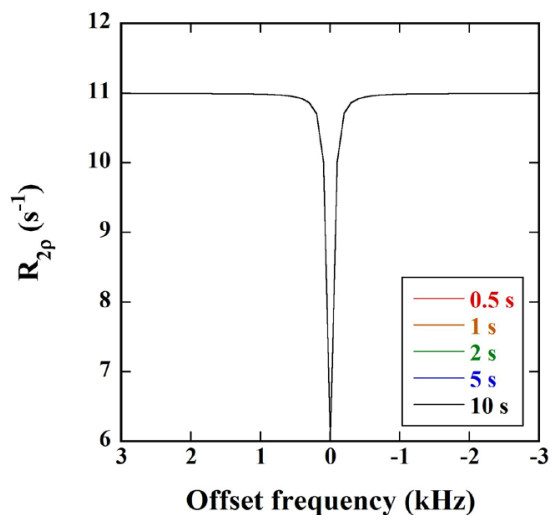

(b)

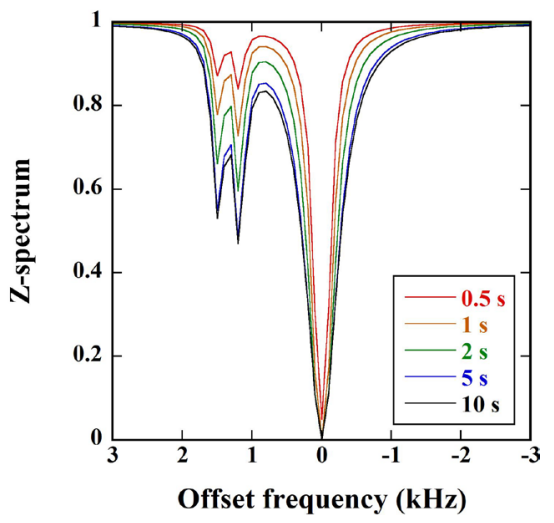

(c)

Figure 4. (a) $R_{1 \rho}$, (b) $R_{2 \rho}$, and (c) Z-spectra as a function of offset frequency $\left(\Delta \omega_{\text {off }}\right)$ for various saturation times $(0.5,1,2,5$, and $10 \mathrm{~s}$ ) in the linear three-pool chemical exchange model (Figure 2(a)). In these simulations, $\omega_{1}, M_{0}^{a}, M_{0}^{b}$, and $M_{0}^{c}$ were assumed to be $50 \mathrm{~Hz}, 1,1 / 250$, and 1/500, respectively. $k_{a b}+k_{b a}$ and $k_{a c}+k_{c a}$ were assumed to be $100 \mathrm{~Hz}$ and $300 \mathrm{~Hz}$, respectively. The values of other parameters such as $T_{1}^{a}\left(=1 / R_{1}^{a}\right)$ are described in the "Simulation Studies" section. Note that all the data with different saturation times overlap in (a) and (b). 


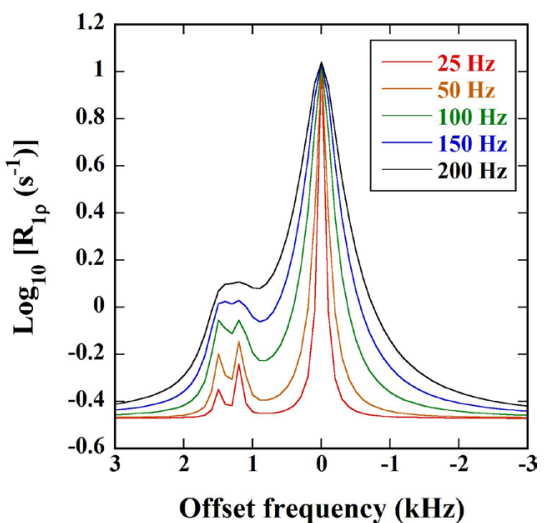

(a)

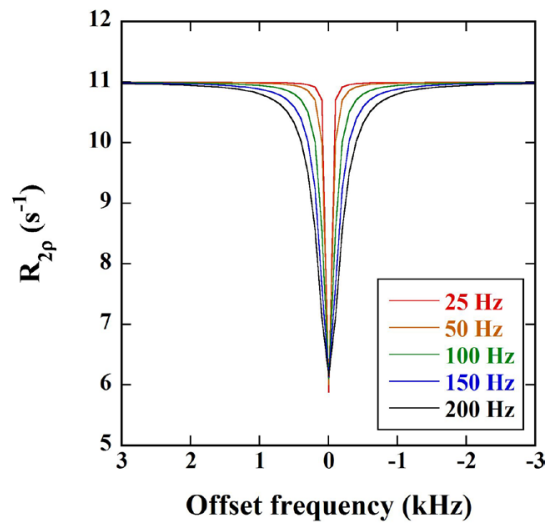

(b)

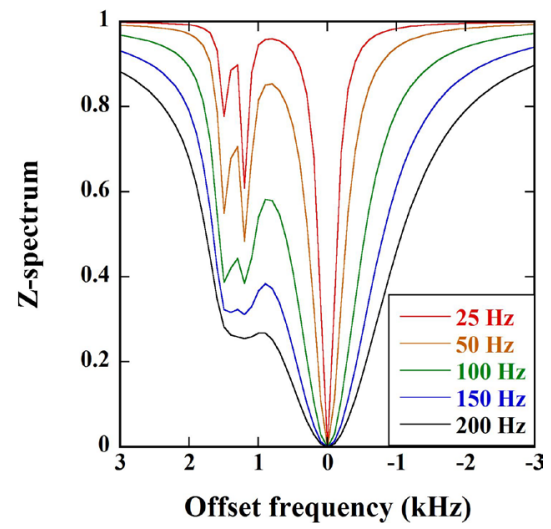

(c)

Figure 5. (a) $R_{1 \rho}$, (b) $R_{2 \rho}$, and (c) Z-spectra as a function of offset frequency $\left(\Delta \omega_{\text {off }}\right)$ for various $\omega_{1}$ values $(25,50,100,150$, and $200 \mathrm{~Hz}$ ) in the linear three-pool chemical exchange model (Figure 2(a)). In these simulations, saturation time, $M_{0}^{a}, M_{0}^{b}$, and $M_{0}^{c}$ were assumed to be $5 \mathrm{~s}, 1,1 / 250$, and $1 / 500$, respectively. $k_{a b}+k_{b a}$ and $k_{a c}+k_{c a}$ were assumed to be $100 \mathrm{~Hz}$ and 300 $\mathrm{Hz}$, respectively. The values of other parameters are described in the "Simulation Studies" section.

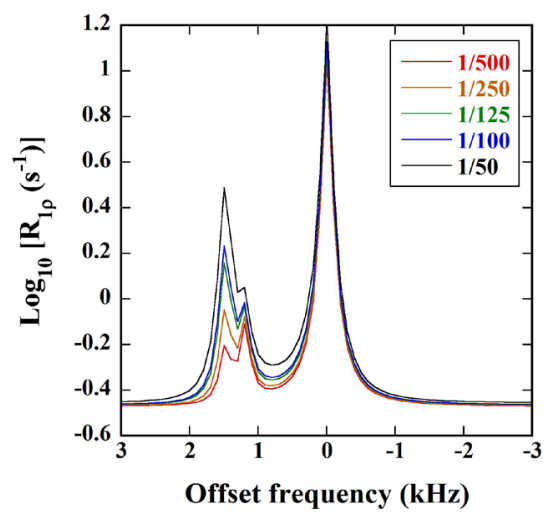

(a)

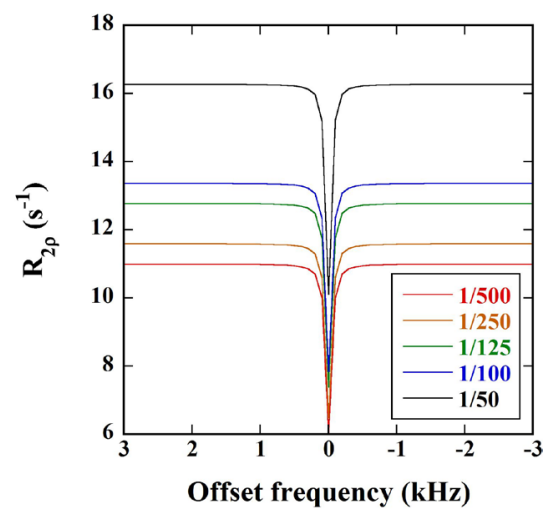

(b)

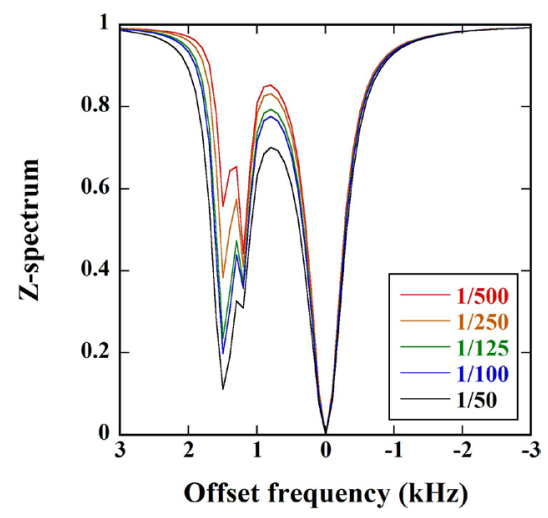

(c)

Figure 6. (a) $R_{1 \rho}$, (b) $R_{2 \rho}$, and (c) Z-spectra as a function of offset frequency $\left(\Delta \omega_{\text {off }}\right)$ for various $M_{0}^{c}$ values $(1 / 500,1 / 250$, $1 / 125,1 / 100$, and $1 / 50$ ) in the triangular three-pool chemical exchange model (Figure 2(b)). In these simulations, saturation time, $\omega_{1}, M_{0}^{a}$, and $M_{0}^{b}$ were assumed to be $5 \mathrm{~s}, 50 \mathrm{~Hz}, 1$, and $1 / 250$, respectively. $k_{a b}+k_{b a}, k_{a c}+k_{c a}$, and $k_{b c}+k_{c b}$ were assumed to be $100 \mathrm{~Hz}, 300 \mathrm{~Hz}$, and $100 \mathrm{~Hz}$, respectively. The values of other parameters are described in the "Simulation Studies" section.

and $-1043.7 \mathrm{~Hz}(-3.5 \mathrm{ppm})$ in the $R_{1 \rho}$ and Z-spectrum plots. Note that these peaks derive from pool $A$, pool $B$, pool $C$, and pool $D$, respectively. The distinct separation among these peaks degraded with increasing $\omega_{1}$ value.

Figure 8 shows the $R_{1 \rho}$ (a), $R_{2 \rho}$ (b), and Z-spectra (c) as a function of $\Delta \omega_{\text {off }}$ for various $M_{0}^{d}$ values $(1 / 500,1 / 250,1 / 125,1 / 100$, and $1 / 50)$ in the star-type four-pool chemical exchange model (Figure 3(b)). As in Figure 7, the peaks at $0 \mathrm{~Hz}(0 \mathrm{ppm}), 1192.8 \mathrm{~Hz}(4 \mathrm{ppm}), 1491.0 \mathrm{~Hz}(5 \mathrm{ppm})$, and $-1043.7 \mathrm{~Hz}$ (-3.5 ppm) derive from pool $A$, pool $B$, pool $C$, and pool $D$, respectively. As shown in Figure 8(a) and Figure 8(c), the peaks of $R_{1 \rho}$ and Z-spectra at $-1043.7 \mathrm{~Hz}(-3.5 \mathrm{ppm})$ derived from pool $D$ changed depending on the $M_{0}^{d}$ value. The $R_{2 \rho}$ values slightly increased with increasing $M_{0}^{d}$ value (Figure 8(b)). 


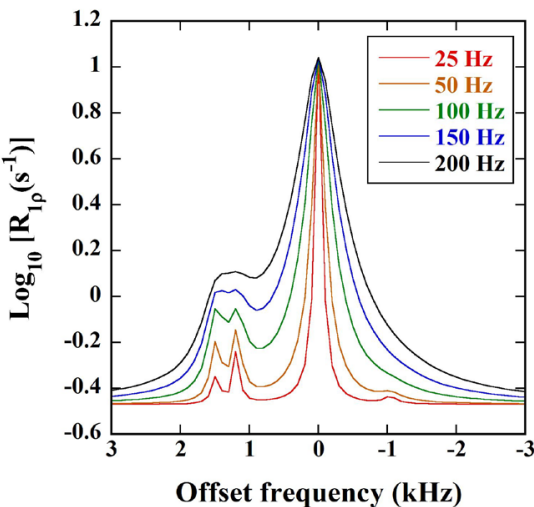

(a)

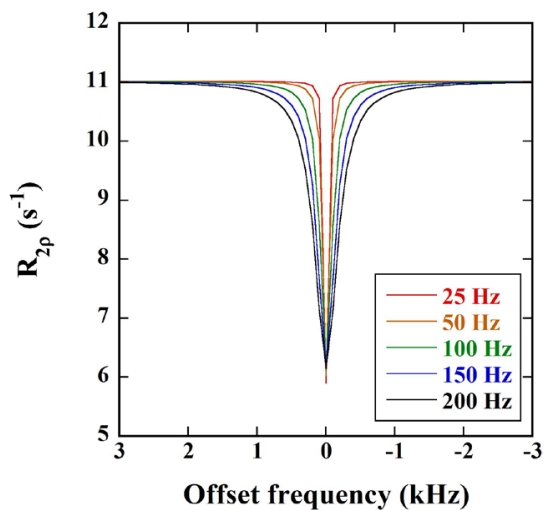

(b)

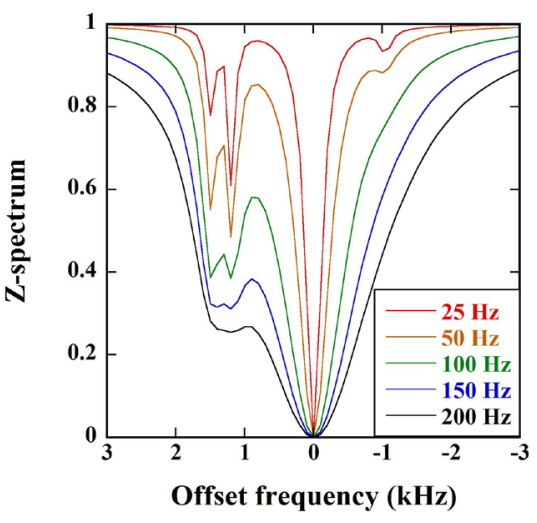

(c)

Figure 7. (a) $R_{1 \rho}$, (b) $R_{2 \rho}$, and (c) Z-spectra as a function of offset frequency $\left(\Delta \omega_{\text {off }}\right)$ for various $\omega_{1}$ values $(25,50,100,150$, and $200 \mathrm{~Hz}$ ) in the linear four-pool chemical exchange model (Figure 3(a)). In these simulations, saturation time, $M_{0}^{a}, M_{0}^{b}$, $M_{0}^{c}$, and $M_{0}^{d}$ were assumed to be $5 \mathrm{~s}, 1,1 / 250,1 / 500$, and $1 / 500$, respectively. $k_{a b}+k_{b a}, k_{a c}+k_{c a}$, and $k_{a d}+k_{d a}$ were assumed to be $100 \mathrm{~Hz}, 300 \mathrm{~Hz}$, and $10 \mathrm{~Hz}$, respectively. The values of other parameters are described in the "Simulation Studies" section.

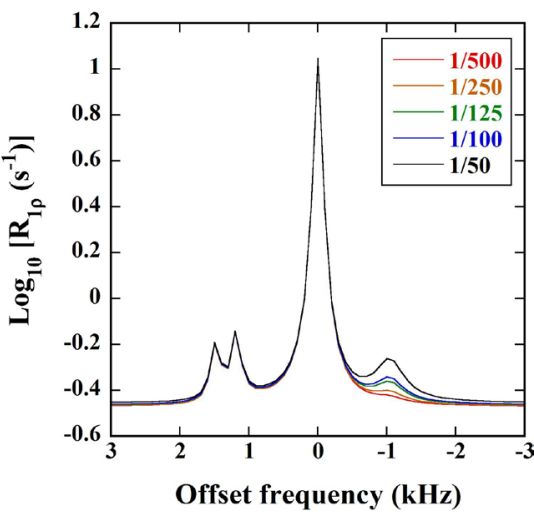

(a)

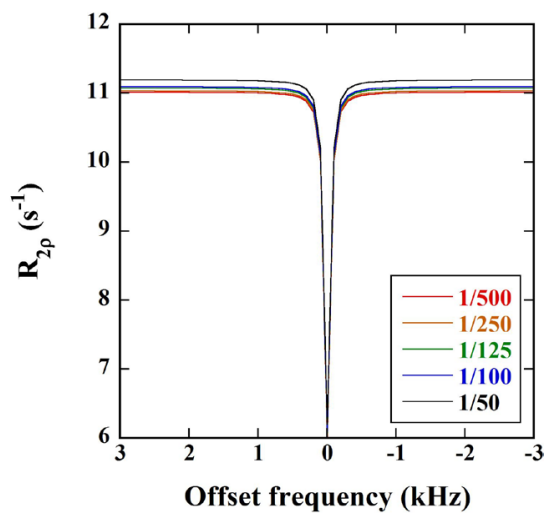

(b)

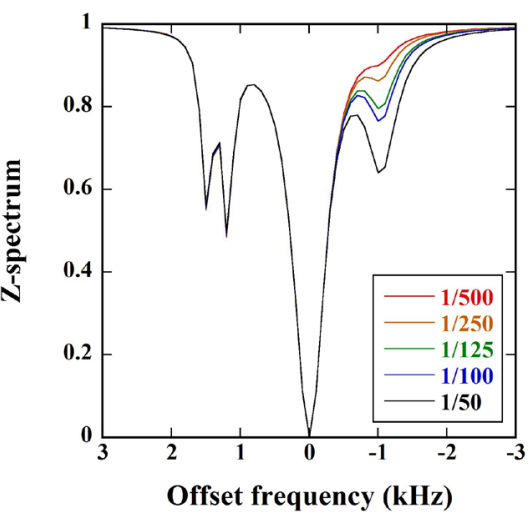

(c)

Figure 8. (a) $R_{1 \rho}$, (b) $R_{2 \rho}$, and (c) Z-spectra as a function of offset frequency $\left(\Delta \omega_{\text {off }}\right)$ for various $M_{0}^{d}$ values $(1 / 500,1 / 250$, $1 / 125,1 / 100$, and $1 / 50$ ) in the star-type four-pool chemical exchange model (Figure $3(\mathrm{~b})$ ). In these simulations, saturation time, $\omega_{1}, M_{0}^{a}, M_{0}^{b}$, and $M_{0}^{c}$ were assumed to be $5 \mathrm{~s}, 50 \mathrm{~Hz}, 1,1 / 250$, and $1 / 500$, respectively. $k_{a b}+k_{b a}, k_{a c}+k_{c a}$, and $k_{a d}+k_{d a}$ were assumed to be $100 \mathrm{~Hz}, 300 \mathrm{~Hz}$, and $10 \mathrm{~Hz}$, respectively. The values of other parameters are described in the "Simulation Studies" section.

Figure 9 shows the $R_{1 \rho}$ (a), $R_{2 \rho}$ (b), and Z-spectra (c) as a function of $\Delta \omega_{\text {off }}$ for various $k_{c d}+k_{d c}$ values $(10,100,200,500$, and $1000 \mathrm{~Hz})$ in the kitetype four-pool chemical exchange model (Figure 3(c)). As in Figure 7 and Figure 8, the peaks at $0 \mathrm{~Hz}(0 \mathrm{ppm}), 1192.8 \mathrm{~Hz}(4 \mathrm{ppm}), 1491.0 \mathrm{~Hz}(5 \mathrm{ppm})$, and $-1043.7 \mathrm{~Hz}(-3.5 \mathrm{ppm})$ correspond to pool $A$, pool $B$, pool $C$, and pool $D$, respectively. As shown in Figure 9(a) and Figure 9(c), the peaks of $R_{1 \rho}$ and Z-spectra at $-1043.7 \mathrm{~Hz}(-3.5 \mathrm{ppm})$ changed depending on the $k_{c d}+k_{d c}$ value. The peaks at $1491.0 \mathrm{~Hz}(5 \mathrm{ppm})$ derived from pool $C$ also changed but to a lesser extent. The $R_{2 \rho}$ value did not change depending on the $k_{c d}+k_{d c}$ value (Figure 9(c)).

\section{Discussion}

In this study, we developed a simple equation for solving the time-dependent 


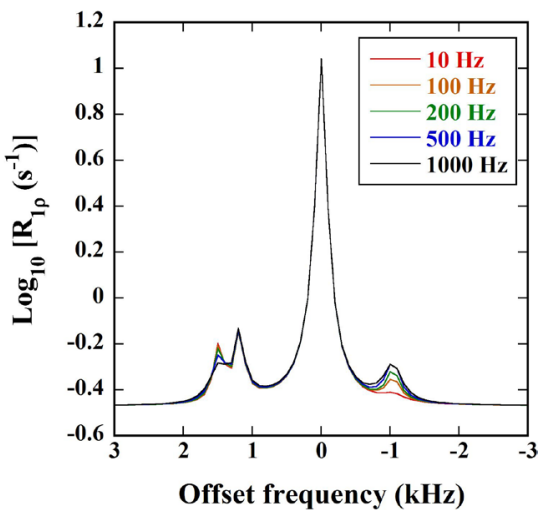

(a)

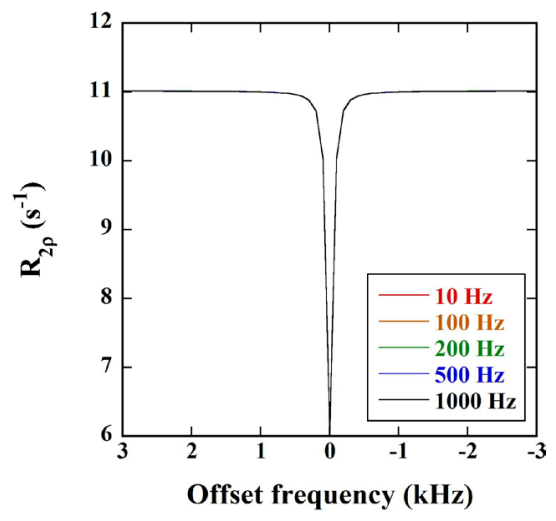

(b)

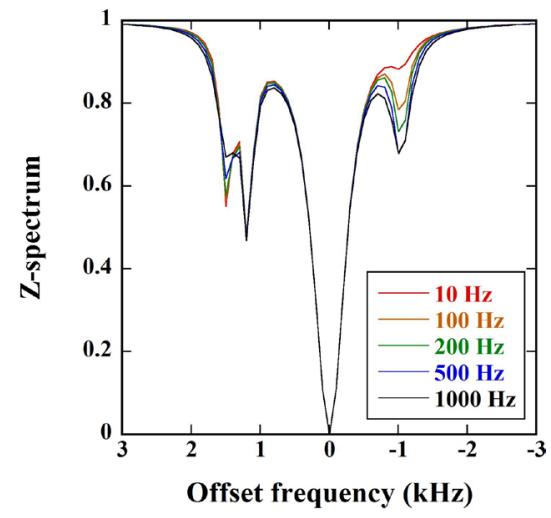

(c)

Figure 9. (a) $R_{1 \rho}$, (b) $R_{2 \rho}$, and (c) Z-spectra as a function of offset frequency $\left(\Delta \omega_{\text {off }}\right)$ for various $k_{c d}+k_{d c}$ values (10, 100, 200, 500, and $1000 \mathrm{~Hz}$ ) in the kite-type four-pool chemical exchange model (Figure 3(c)). In these simulations, saturation time, $\omega_{1}, M_{0}^{a}, M_{0}^{b}, M_{0}^{c}$, and $M_{0}^{d}$ were assumed to be $5 \mathrm{~s}, 50 \mathrm{~Hz}, 1,1 / 250,1 / 500$, and 1/500, respectively. $k_{a b}+k_{b a}, k_{a c}+k_{c a}$, and $k_{a d}+k_{d a}$ were assumed to be $100 \mathrm{~Hz}, 300 \mathrm{~Hz}$, and $10 \mathrm{~Hz}$, respectively. The values of other parameters are described in the "Simulation Studies" section.

Bloch-McConnell equations in the presence of multiple chemical exchange pools by combining our previous method [11] and the approach presented by Koss et al. [12]. As described in our previous paper [11], the numerical solutions obtained by our method agreed with the analytical solutions given by Mulkern and Williams [14]. We also compared the solutions obtained by our method for the two-pool exchange model with those obtained using a fourth/fifth-order RungeKutta-Fehlberg (RKF) algorithm and found that there was a good agreement between them [11]. These results appear to indicate the validity of our method. Furthermore, the computation time was considerably reduced when using our method (by a factor of approximately 2500 compared to the case when using the RKF algorithm [11]). Thus, our method can be included in the nonlinear leastsquares fitting routine to calculate parameters such as the exchange rate or lifetime of CEST agents [10].

For calculating the solutions to the time-dependent Bloch-McConnell equations using Equation (12), most computation time is spent calculating the eigenvectors and eigenvalues of matrix $\boldsymbol{A}$. However, it is necessary to carry out this calculation only once regardless of $t$ in Equation (12). As previously described, in this study, the matrix exponential was computed using the MATLAB ${ }^{\circ}$ function "expm", in which a scaling and squaring algorithm with Pade approximation [15] has been used.

In our previous study [11], we used the two-pool exchange model for CEST or APT MRI as an illustrative example. As pointed out by Woessner et al. [10], paramagnetic CEST agents often have more than one type of exchangeable proton. For such cases, it is necessary to expand the Bloch-McConnell equations to multi-pool exchange models. Recently, Koss et al. [12] presented a generalized expression for the evolution matrix in the Bloch-McConnell equations in the presence of multiple chemical exchange sites. In their method, the Kronecker tensor 
product was used [12]. As shown in this study, our method could be easily extended to multi-pool chemical exchange models by modifying matrix $A$ in Equation (12) with use of their approach.

As previously described, $R_{1 \rho}$ was obtained from the negative of the largest (least negative) real eigenvalue of matrix $A$ in Equation (2). $R_{2 \rho}$ was obtained from the absolute value of the largest real part of the complex eigenvalue of matrix $\boldsymbol{A}$ in Equation (2). We previously compared the $R_{1 \rho}$ and $R_{2 \rho}$ values thus obtained with those obtained numerically and found that there was a good agreement between them [13], indicating the validity of these procedures.

The spectral dependence of CEST is determined by sweeping the RF irradiation frequency while monitoring the water resonance [1]. As previously described, the CEST effect has usually been analyzed using the so-called Z-spectrum [11]. The Z-spectrum is obtained by plotting the $z$ component of the magnetization of pool $A$, i.e., bulk water proton $\left(M_{z}^{a}\right)$ in the form of $M_{z}^{a}$ versus irradiation offset frequency $\left(\Delta \omega_{\text {off }}\right)$ (Equation (24)). As shown in Figure 4(a) and Figure 4(b), $R_{1 \rho}$ and $R_{2 \rho}$ were not affected by the saturation time, because the matrix $\boldsymbol{A}$ in Equation (2) is independent of the saturation time. On the other hand, the Z-spectra were affected by the saturation time, i.e., the CEST effect observed in the Z-spectra increased and saturated with increasing saturation time (Figure $4(\mathrm{c}))$. When the RF irradiation power $\left(\omega_{1}\right)$ was varied, the CEST effect increased with increasing $\omega_{1}$ in $R_{1 \rho}, R_{2 \rho}$, and Z-spectra (Figure 5 and Figure 7). When $\omega_{1}$ is large, however, its saturation bandwidth is broad and thus may directly saturate the bulk water, causing the so-called spillover effect [8]. As shown in Figure 5 and Figure 7, the separation among peaks in the $R_{1 \rho}$ and Z-spectrum plots degraded with increasing $\omega_{1}$, which appears to be due to the spillover effect. These results suggest that $\omega_{1}$ must be determined in consideration of both the CEST effect and the spillover effect. Simulation studies with use of our method will be useful especially in such a case.

As shown in Figure 6(b) and Figure 8(b), the $R_{2 \rho}$ values increased with increasing $M_{0}^{c}$ or $M_{0}^{d}$ value. When $M_{0}^{c}$ or $M_{0}^{d}$ increased, the interaction between bulk water protons and protons in pool $C$ or pool $D$ would increase, leading to an increase of $R_{2}^{a}$. Our previous study demonstrated that the $R_{2 \rho}$ value increased with increasing $R_{2}^{a}$ value in a two-pool chemical exchange model [13]. This would also be applicable to the case of multi-pool chemical exchange models. Thus, the above finding would be able to be explained by the fact that the $R_{2}^{a}$ value increases with increasing $M_{0}^{c}$ or $M_{0}^{d}$ value.

Recently, Koss et al. [12] presented analytical expressions for $R_{1 \rho}$ in the presence of multiple chemical exchange sites and pointed out that analytical solutions facilitate understanding of the relationship between model parameters and the phenomenological relaxation rate constant and can lead to new methodological advances. Numerical solutions also appear to be useful for optimizing parameters such as the saturation time and $\omega_{1}$ for acquiring CEST or APT MRI data, because they can be easily obtained under various and/or complex study conditions in which analytical solutions may not always be obtained. 


\section{Conclusion}

We presented a simple and fast numerical method for solving the time-dependent Bloch-McConnell equations in the presence of multiple chemical exchange pools by combining our previous method [11] and the approach presented by Koss et al. [12]. The present method will be useful for analyzing the complex CEST contrast mechanism and for investigating the optimal conditions for CEST MRI in the presence of multiple chemical exchange pools.

\section{Acknowledgements}

This work was supported in part by a Grant-in-Aid for Challenging Exploratory Research (Grant No. 25670532) from the Japan Society for the Promotion of Science.

\section{References}

[1] Ward, K., Aletras, A. and Balaban, R. (2000) A New Class of Contrast Agents for MRI Based on Proton Chemical Exchange Dependent Saturation Transfer (CEST). Journal of Magnetic Resonance, 143, 79-87. https://doi.org/10.1006/jmre.1999.1956

[2] Goffeney, N., Bulte, J.W.M., Duyn, J., Bryant, L.H. and van Zijl, P.C.M. (2001) Sensitive NMR Detection of Cationic-Polymer-Based Gene Delivery Systems Using Saturation Transfer via Proton Exchange. Journal of American Chemical Society, 123, 8628-8629. https://doi.org/10.1021/ja0158455

[3] Aime, S., Barge, A., Castelli, D.D., Fedeli, F., Mortillaro, A., Nielsen, F.U. and Terreno, E. (2002) Paramagnetic Lanthanide (III) Complexes as pH-Sensitive Chemical Exchange Saturation Transfer (CEST) Contrast Agents for MRI Applications. Magnetic Resonance in Medicine, 47, 639-648. https://doi.org/10.1002/mrm.10106

[4] Snoussi, K., Bulte, J.W.M., Gueron, M. and van Zijl, P.C.M. (2003) Sensitive CEST Agents Based on Nucleic Acid Imino Proton Exchange: Detection of Poly(rU) and of a Dendrimer-Poly(rU) Model for Nucleic Acid Delivery and Pharmacology. Magnetic Resonance in Medicine, 49, 998-1005. https://doi.org/10.1002/mrm.10463

[5] Zhou, J., Lal, B., Wilson, D.A., Laterra, J. and van Zijl, P.C.M. (2003) Amide Proton Transfer (APT) Contrast for Imaging of Brain Tumors. Magnetic Resonance in Medicine, 50, 1120-1126. https://doi.org/10.1002/mrm.10651

[6] Sun, P.Z., Murata, Y., Lu, J., Wang, X., Lo, E.H. and Sorensen, A.G. (2008) Relaxation-Compensated Fast Multislice Amide Proton Transfer (APT) Imaging of Acute Ischemic Stroke. Magnetic Resonance in Medicine, 59, 1175-1182.

https://doi.org/10.1002/mrm.21591

[7] Maruyama, S., Ueda, J., Kimura, A. and Murase, K. (2016) Development and Characterization of Novel LipoCEST Agents Based on Thermosensitive Liposomes. Magnetic Resonance in Medical Sciences, 15, 324-334. https://doi.org/10.2463/mrms.mp.2015-0039

[8] Sun, P.Z. (2010) Simultaneous Determination of Labile Proton Concentration and Exchange Rate Utilizing Optimal RF Power: Radio Frequency Power (RFP) Dependence of Chemical Exchange Saturation Transfer (CEST) MRI. Journal of Magnetic Resonance, 202, 155-161. https://doi.org/10.1016/j.jmr.2009.10.012

[9] Sun, P.Z. (2010) Simplified and Scalable Numerical Solution for Describing Multi-Pool Chemical Exchange Saturation Transfer (CEST) MRI Contrast. Journal of Magnetic Resonance, 205, 235-241. https://doi.org/10.1016/j.jmr.2010.05.004 
[10] Woessner, D.E., Zhang, S., Merritt, M.E. and Sherry, A.D. (2005) Numerical Solution of the Bloch Equations Provides Insights into the Optimum Design of PARACEST Agents for MRI. Magnetic Resonance in Medicine, 53, 790-799. https://doi.org/10.1002/mrm.20408

[11] Murase, K. and Tanki, N. (2011) Numerical Solutions to the Time-Dependent Bloch Equations Revisited. Magnetic Resonance Imaging, 29, 126-131. https://doi.org/10.1016/j.mri.2010.07.003

[12] Koss, H., Rance, M. and Palmer, A.G. (2017) General Expression for $R_{1 \rho}$ Relaxation for $N$-Site Chemical Exchange and the Special Case of Linear Chains. Journal of Magnetic Resonance, 274, 36-45. https://doi.org/10.1016/j.jmr.2016.10.015

[13] Murase, K. (2013) A Theoretical and Numerical Consideration of the Longitudinal and Transverse Relaxations in the Rotating Frame. Magnetic Resonance Imaging, 31, 1544-1558. https://doi.org/10.1016/j.mri.2013.07.004

[14] Mulkern, R.V. and Williams, M.L. (1993) The General Solution to the Bloch Equation with Constant RF and Relaxation Terms: Application to Saturation and Slice Selection. Medical Physics, 20, 5-13. https://doi.org/10.1118/1.597063

[15] Higham, N.J. (2005) The Scaling and Squaring Method for the Matrix Exponential Revisited. SIAM Journal on Matrix Analysis and Applications, 26, 1179-1193. https://doi.org/10.1137/04061101X

Submit or recommend next manuscript to SCIRP and we will provide best service for you:

Accepting pre-submission inquiries through Email, Facebook, LinkedIn, Twitter, etc. A wide selection of journals (inclusive of 9 subjects, more than 200 journals) Providing 24-hour high-quality service User-friendly online submission system Fair and swift peer-review system Efficient typesetting and proofreading procedure Display of the result of downloads and visits, as well as the number of cited articles Maximum dissemination of your research work

Submit your manuscript at: http://papersubmission.scirp.org/ Or contact ojapps@scirp.org 